\title{
Asymmetries in single and triple hop are not detrimental to change of direction speed
}

\author{
Thomas Dos'Santos, Christopher Thomas, Paul A. Jones, Paul Comfort
}

\begin{abstract}
Objectives: To determine the impact of between limb asymmetries in hop performance on change of direction speed (CODS).
Design and Methods: Twenty-two multisport collegiate athletes (mean \pm SD; age: $21.8 \pm 3.4$ years, height: $178.1 \pm 6.7 \mathrm{~cm}$, mass: $73.5 \pm 7.1 \mathrm{~kg}$ ) performed three single and triple horizontal hops for distance per limb, followed by three modified 505 and $90^{\circ}$ cut CODS trials each side to establish imbalances between right and left, and dominant (D) and non-dominant (ND) limbs. Limb dominance was defined as the limb that produced the furthest hop or faster CODS performance.

Results: Paired sample t-tests revealed no significant differences in hop performance and CODS performance between right and left limbs $(p>0.05, g \leq 0.11)$, however, significant differences were observed when comparing D and ND limbs $(p<0.001$, $g=0.46-0.61)$. No significant correlations were observed between hop imbalance and CODS performance $(p>0.05, \mathrm{r} \leq 0.35)$. Low agreements $(32-55 \%)$ were demonstrated between like for like identifications of asymmetry for CODS and hop performance.

Conclusions: Imbalances in hop and CODS were present; however, greater hop imbalances were not detrimental to CODS. Furthermore, the D limb for hopping did not necessarily correspond to faster performance from that limb during $180^{\circ}$ turns and $90^{\circ}$ cuts (plant foot). Collegiate male multi-sport athletes with imbalances within the range reported within this study $(\leq 15 \%)$ should not experience associated CODS detriments.
\end{abstract}

(Journal of Trainology 2017;6:35-41)

Key words: imbalance $\boldsymbol{\square}$ limb dominance $\boldsymbol{\square}$ performance deficit

\section{INTRODUCTION}

A strength discrepancy of $\geq 10-15 \%$ between sides is considered to represent a potentially problematic asymmetry. ${ }^{1}$ Greater limb asymmetries are suggested to place an athlete at an increased risk of injury, ${ }^{2-4}$ conversely researchers have refuted this connection between strength asymmetries and injury $^{5,6}$. Furthermore, contradictory findings have been reported with investigations revealing a negative impact on athletic performance, ${ }^{7-9}$ whereas no negative effect on athletic performance has also been demonstrated ${ }^{9-11}$.

Between limb asymmetries are commonly assessed via isokinetic dynamometry, ${ }^{4,5}, 12$ unilateral jump tasks, ${ }^{7,13,14}$ and the unilateral isometric mid-thigh pull ${ }^{15}$. However, the abovementioned assessments require expensive equipment which are not readily available to strength and conditioning and rehabilitation practitioners and involve extensive periods of time to collect and analyse data. Alternatively, single leg hops for distance (SLH) are easier to administer and are cost effective, producing highly reliable measures of lower limb triple extension function, ${ }^{16-18}$ while able to detect asymmetries ${ }^{12,17}$. Furthermore, this assessment can be used in the field as an indirect measure of unilateral lower body horizontal power, force propulsion and acceptance, which can be used to provide rehabilitation and training information. ${ }^{19}$

The ability to change direction is a key component of multidirectional sports and is influenced by technical, speed and lower limb strength and power qualities. ${ }^{8,20}$ Imbalances between limbs has been stated to be a component of CODS. ${ }^{21}$ Theoretically, being equally proficient in force production would be advantageous for the braking and propulsive requirements of change of direction speed (CODS), however it is inconclusive whether strength asymmetries negatively impact CODS. Previous studies have shown imbalances in unilateral vertical drop jump height and reactive strength, ${ }^{7,8}$ and eccentric hamstring torque 9 were associated with slower CODS. Conversely, no detrimental impact in CODS was reported for imbalances in unilateral vertical jump power, ${ }^{11}$ while faster CODS athletes demonstrated significantly greater imbalances in knee extensor torque ${ }^{9}$. The lack of consensus may be explained by differences in the methods to assess imbalances, CODS tasks, angle of direction change, asymmetry calculation, statistical analysis procedures and subject populations.

Strong inverse relationships have been observed between SLH and triple hops for distance (SLTH) with CODS $^{10,18,22}$ attributed to the similarities in the force vector and push off mechanism ${ }^{16}$. Conversely, there is a paucity of research investigating the influence of between limb asymmetries from horizontal hops for distance on CODS. To our knowledge only one study has investigated the influence of between limb asymmetries in unilateral hops and jumps for distance on CODS, demonstrating no detrimental effect on CODS. ${ }^{10}$ Further evidence is required to improve our understanding regarding the effect of between limb asymmetries on CODS using assessments that

Received March 18, 2017; accepted June 17, 2017

From the Directorate of Sport, Exercise and Physiotherapy, University of Salford, Salford, Greater Manchester, United Kingdom (T.D., C.T., P.A.J., P.C.) Communicated by Takashi Abe, PhD

Correspondence to Mr. Thomas Dos'Santos, C702, Allerton Building, Frederick Road Campus, Salford, Greater Manchester, United Kingdom, M6 6PU. Email: t.dossantos@edu.salford.ac.uk

Journal of Trainology 2017;6:35-41 @2012 The Active Aging Research Center http://trainology.org/ 
are readily available to practitioners in the sporting field. Moreover, it remains unclear whether athletes with lower limbs asymmetries in horizontal hop performance also demonstrate superior performance from that same limb (push off limb) during turning and cutting tasks.

The aims of this study were to: 1) compare SLH and SLTH between right and left, and dominant (D) and non-dominant (ND) limbs, and to determine if significant differences and imbalances were present between limbs in collegiate multisport athletes. 2) explore the relationship between the size of imbalances in SLH and SLTH distances and CODS. 3) determine if collegiate athletes exhibit directional dominance during CODS and 4) investigate if athletes with hop asymmetries perform faster from that push off limb during CODS. It was hypothesised that no significant differences would be found between comparisons of right and left limbs for SLH and SLTH and CODS directions; however, significant differences would be found when comparing D to ND. It was additionally hypothesised that greater asymmetries in hop distance would result in slower CODS performance.

\section{METHODS \\ Experimental Approach to the problem}

This study compared SLH and SLTH between right and left, and D and ND limbs and examined the relationship of between limb asymmetries in hop distance with modified $505(\bmod 505)$ and $8 \mathrm{~m} \mathrm{90}$ cut CODS performance. A cross-sectional analysis of collegiate team sport athletes was conducted; Pearson's correlation analysis was performed to determine if significant relationships were present for hop distance imbalances with CODS, similar to previous research.,10 Paired sample t-tests compared left and right limbs and D and ND for SLH, SLTH and CODS to determine if any between limb differences were present, similar to previous imbalance research. ${ }^{12,23}$ Percentage agreements between like for like classifications were performed to determine if asymmetrical limbs corresponded to directional dominance during the $\bmod 505$ and $90^{\circ}$ cut. $^{24}$

\section{Subjects}

Twenty-two male collegiate team sport athletes (soccer $\mathrm{n}=$ 10 , rugby $\mathrm{n}=6$ and cricket $\mathrm{n}=6$ ) participated in this study (mean \pm SD; age: $21.8 \pm 3.4$ years, height: $178.1 \pm 6.7 \mathrm{~cm}$, mass: $73.5 \pm 7.1 \mathrm{~kg}$ ). All subjects were right hand D (throwing), and 20 were right leg D (kicking). A minimum sample size of 19 subjects was determined from an a priori power analysis using $\mathrm{G}^{*}$ Power (Version 3.1, University of Dusseldorf, Germany) ${ }^{25}$ based upon squared multiple correlation of 0.36 (value of maximum prediction coefficient reported in literature for similar studies ${ }^{7}$ ), a power of 0.8 and type 1 error or alpha level of $<0.05$. All subjects had a minimum one-year resistance training experience and were free from lower limb injuries six months prior to testing. All subjects were instructed to wear appropriate clothing and footwear, not have consumed alcohol 24 hours or caffeine two hours prior to testing, to maintain their normal diet and refrain from training 48 hours prior to the testing session. Approval for the study was provided by the University's ethics committee and all subjects pro- vided written consent.

\section{Procedures}

Testing was conducted over one session, and all subjects were familiarised with all assessments due to being regularly performed throughout the season for screening and monitoring purposes. All subjects performed a standardised progressive warm up directed by the investigator which included five minutes of non-fatiguing dynamic stretches, activation and mobilisation exercises including body weight squats and lunges before progressing to 10 minutes jogging, bounding, skipping, light runs and sprints.

\section{Hop Testing}

The SLH and SLTH tests were used as a measure of horizontal jump performance and performed in accordance to previous research. ${ }^{12,17}$ Three warm-up trials were performed on each leg, for both hop tests to control for learning effects. ${ }^{17}$ All hops were performed with hands akimbo to isolate the contribution from the lower limb. ${ }^{16} \mathrm{SLH}$ required subjects to perform a countermovement, then hopped as far forward as possible, taking off from one leg, before landing on the same leg. SLTH involved subjects performing three consecutive maximal hops on the same limb and sticking the landing after the final hop. If the subject did not stick the landing for at least two seconds, or if the subject removed their hands from their waist, the trial was disregarded and another was attempted after one minute of rest. ${ }^{17}$ The distance was measured to the nearest $0.01 \mathrm{~m}$ using a standard tape measure, perpendicular from the front of the start line to the posterior aspect of the heel at landing. Three maximal trials were recorded on each leg for both tests alternating between right and left limbs, with one minute of rest between trials. The best performance of each leg from each hop test was used for further analysis.

\section{Change of Direction Speed Assessments}

All subjects received 10 minutes' rest between hop and CODS tests. CODS were assessed by a $\bmod 505$ and a $90^{\circ}$ cutting task; all performed on an indoor track (Mondo, SportsFlex, 10 mm; Mondo America Inc., Mondo, Summit, NJ, USA). Completion time was measured using Brower timing gates (Draper, UT, USA) placed approximately at hip height for all athletes. All subjects performed six trials for each COD task in an alternating order; three changing direction with a left foot plant, and three changing direction with a right foot plant interspersed with two minutes' rest between trials. Subjects were allowed three practice attempts to familiarise themselves with the movement patterns required.

Mod505 testing involved sprinting to a line marked $5 \mathrm{~m}$ from the start (starting $0.3 \mathrm{~m}$ behind the start line), planting their left or right foot on the line, turn $180^{\circ}$ and sprinting back $5 \mathrm{~m}$ through the finish as fast as possible. ${ }^{26}$ Similarly, the $90^{\circ}$ cutting task involved sprinting forwards $5 \mathrm{~m}$ and performing a $90^{\circ}$ cut left or right, and exiting $3 \mathrm{~m}$ through the finish where timing gates were also placed. If the subject changed direction before hitting the turning line, or changed direction off the incorrect foot, the trial was disregarded and the subject com- 
pleted another trial after the rest period. The fastest completion time for each limb and task was used for further analysis.

\section{Asymmetry Index}

Asymmetry index (imbalance between right and left limbs) was calculated by the formulae (right leg - left leg/ right leg $\times$ 100) for hops and CODS performance. ${ }^{27}$ Limb dominance was defined as the limb that produced the furthest hop or faster CODS performance. ${ }^{12,23}$ Asymmetry index for D and ND limbs was calculated by the formulae (dominant leg - non dominant leg/ dominant leg $\times 100$ ) for hops and CODS performance. ${ }^{12}$

\section{Statistical Analyses}

Mean \pm SD were calculated for all variables. Normality was confirmed for all variables using a Shapiro Wilks-test. Withinsession reliability was assessed via intraclass correlation coefficients (ICC), 95\% confidence intervals (CI) and coefficient of variation $(\mathrm{CV})$ calculated as $\mathrm{SD} /$ mean $\times 100$. Minimum acceptable reliability was determined with an ICC $>0.7$ and $\mathrm{CV}<10 \% .^{28}$ Magnitude of differences between limbs were assessed with paired sample t-tests and effect sizes calculated using Hedges' $g$ method ${ }^{29}$ and interpreted using Hopkins' scale. ${ }^{30}$ Relationships between CODS completion time and D-ND imbalances were analysed using Pearson's productmoment correlation and were Bonferonni corrected to reduce likelihood of type 1 error. Correlations were evaluated using Hopkins' scale. ${ }^{31}$ The criterion for significance was set at $p \leq 0.05$. All statistical analyses were performed using SPSS (version 23, IBM, New York, NY, USA).

To assess the agreement between the D limb for hops and D CODS performance, asymmetry thresholds for each hop parameter were established as mean imbalance $+(0.2 S D$ of the mean) and mean imbalance $-(0.2 S D$ of the mean) for CODS imbalance. ${ }^{10}$ Subjects with imbalances which exceeded the threshold were classified as asymmetrical, imbalances below the threshold were subsequently classified as balanced. The overall level of agreement between like for like asymmetries (or balanced) for CODS and hops were calculated by counting the frequency and percentage of like for like identifications of asymmetry (i.e., either both asymmetrical or both balanced) using the equation (frequency of like for like diagnoses/ number of subjects) $\times 100 .{ }^{24}$ Percentage agreements $\geq$ $80 \%$ were considered good.

\section{RESULTS}

High ICCs and low levels of variance were observed for SLH $(\mathrm{ICC}=0.96-0.97, \mathrm{CV}=3.8-4.1 \%)$, SLTH $(\mathrm{ICC}=0.96-$ $0.97, \mathrm{CV}=3.2-3.4 \%), \bmod 505(\mathrm{ICC}=0.88, \mathrm{CV}=2.4-$ $2.6 \%$ ) and $90^{\circ}$ cut performance ( $\mathrm{ICC}=0.88-0.92, \mathrm{CV}=2.6-$ $2.8 \%$ ); all meeting minimum acceptable reliability criteria.

No significant differences were observed between right and left limbs for hopping and CODS (Table 1) $(p>0.05$, ES $\leq$ 0.11 ). However, directional dominance was observed for both CODS tasks $(p<0.0001, g=-0.49$ to -0.61$)$ and small significant differences were demonstrated between D and ND hopping performance (Table 1) $(p<0.0001, g=0.46-0.50)$. No significant correlations were observed between hop imbalances and CODS (Table 2) $(\mathrm{r} \leq 0.35, \mathrm{p}>0.05)$, and hop imbalance with CODS imbalance $(r \leq 0.11, p>0.05$.)

Agreement between like for like identifications (including asymmetrical or balanced) are presented in Table 3 along with frequency of asymmetry classification. Low agreements were observed between hop and CODS asymmetry (41-55\%). In addition, poor agreement was demonstrated between mod505 and cutting like for like identification asymmetry (32\%). Eight and nine subjects demonstrated imbalances greater than asymmetry thresholds -2.74 and $-4.93 \%$ for $\bmod 505$ and $90^{\circ}$ cut performance, respectively (Table 3; Figures 1). Eight subjects

Table 1 Right vs Left and Dominant vs Non-Dominant Limb Comparisons for Hops and CODS Performance

\begin{tabular}{|c|c|c|c|c|c|c|c|c|c|c|c|c|c|c|c|c|c|}
\hline \multirow{2}{*}{ Variable } & \multicolumn{2}{|c|}{ Right } & \multicolumn{2}{|c|}{ Left } & \multicolumn{2}{|c|}{ Imbalance (\%) } & & \multirow{2}{*}{$\alpha$} & \multicolumn{2}{|c|}{ Dominant } & \multicolumn{2}{|c|}{ Non-Dominant } & \multicolumn{2}{|c|}{ Imbalance (\%) } & & \multirow{2}{*}{$\sigma$} & \multirow{2}{*}{$\begin{array}{c}\text { Asymmetry } \\
\text { Threshold } \\
(\%)\end{array}$} \\
\hline & Mean & SD & Iean & D & Mean & SD & & & Mean & SD & Mean & SD & Mean & SD & & & \\
\hline SLH (m) & 1.56 & 0.20 & 1.57 & 0.23 & -0.48 & 8.41 & 22 & -0.03 & 1.62 & 0. & & 0. & 6.25 & 4.99 & $<0.0$ & 0.46 & 7.25 \\
\hline SLTH (m) & 5.11 & 0.59 & 5.10 & 0.65 & 0.06 & 7.13 & 0.891 & 0.01 & 5.26 & 0.62 & 4.95 & 0.58 & 5.69 & 3.63 & $<0.0001$ & 0.50 & 6.42 \\
\hline $\bmod 505(s)$ & 2.68 & 0.14 & 2.66 & 0.12 & 0.46 & 2.97 & 0.42 & 0.11 & 2.64 & 0.12 & 2.70 & 0.13 & -2.34 & 1.98 & $<0.0001$ & -0.49 & -2.74 \\
\hline $90^{\circ}$ Cut (s) & 1.96 & 0.12 & 1.97 & 0.15 & -0.54 & 5.36 & 0.66 & -0.07 & 1.92 & 0.13 & 2.00 & 0.14 & -4.27 & 3.28 & $<0.0001$ & -0.61 & -4.93 \\
\hline
\end{tabular}

Key: SLH = single-leg hop; SLTH = single-leg triple hop; $\bmod 505=$ Modified505

Table 2 Pearson's Product-Moment Correlations Between Hop Imbalances and CODS

\begin{tabular}{|c|c|c|c|c|c|c|c|c|c|c|}
\hline D to ND & $\bmod 505 \mathrm{~L}$ & $\bmod 505 R$ & $90^{\circ}$ Cut L & $90^{\circ}$ Cut R & $\bmod 505 \mathrm{D}$ & $\bmod 505$ ND & $90^{\circ}$ Cut D & $90^{\circ}$ Cut ND & $\begin{array}{c}\text { mod505 } \\
\text { Imbalance }\end{array}$ & $\begin{array}{c}90^{\circ} \text { Cut } \\
\text { Imbalance }\end{array}$ \\
\hline & r value & r value & r value & r value & r value & r value & r value & r value & r value & r value \\
\hline SLH & 0.22 & 0.22 & 0.35 & 0.18 & .207 & .242 & .267 & .270 & -0.08 & .04 \\
\hline SLTH & -0.15 & -0.29 & -0.08 & -0.09 & -.231 & -.212 & -.061 & -.109 & -0.04 & 0.11 \\
\hline
\end{tabular}

Key: $\mathrm{R}=$ Right; $\mathrm{L}=\mathrm{Left} ; \mathrm{SLH}=$ single-leg hop; SLTH = single-leg triple hop; $\bmod 505=$ Modified505;D = Dominant; ND = Non-dominant; $* p<0.05$ 
Table 3 Percentage Agreements Between Like for Like Identifications of Asymmetry Classification

\begin{tabular}{cccccc}
\hline & & $\operatorname{mod505}$ & $\mathbf{9 0}^{\circ}$ Cut & SLH & SLTH \\
\cline { 3 - 6 } Frequency (n=) & $\mathrm{B}$ & 14 & 13 & 14 & 12 \\
& $\mathrm{~L}$ & 4 & 4 & 4 & 6 \\
\hline $\begin{array}{c}\text { \% agreement with mod505 performance } \\
\text { (like for like identification) }\end{array}$ & $\mathrm{R}$ & 4 & 5 & 4 & 4 \\
\hline $\begin{array}{c}\text { \% agreement with cutting performance } \\
\text { (like for like identification) }\end{array}$ & & 32 & 55 & 50 \\
\hline
\end{tabular}

SLH = single-leg hop; SLTH = single-leg triple hop; $\mathrm{R}=$ Right Asymmetrical; $\mathrm{L}=$ Left Asymmetrical; $\mathrm{B}=$ Balanced

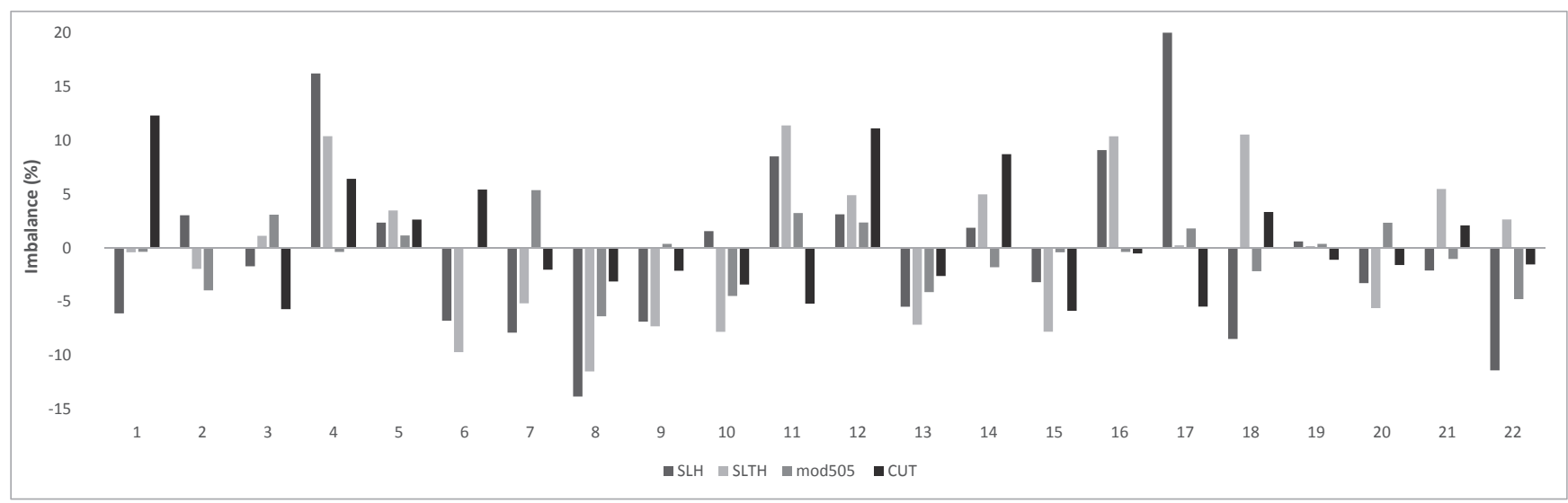

Figure 1 Individual right-left imbalances for SLH and SLTH distance and CODS (Positive imbalance values indicate right limb dominance and negative imbalance values indicate left limb dominance)

were classified as asymmetrical (SLH) with an imbalance exceeding $7.25 \%$ (Table 3; Figure 1), however only two of those subjects demonstrated directional dominance from that same limb for mod505 performance; and only one subject corresponded to faster performance for cutting (Figure 1). Similarly, 10 subjects were classified as asymmetrical in SLTH, but only three subjects demonstrated asymmetries which corresponded to the directional dominance for mod505, and two subjects corresponded to cutting performance (Table 3; Figure 1).

\section{DISCUSSION}

The aims of this study were to determine if there were between limb asymmetries in hopping and CODS, and to explore if greater between limb asymmetries were detrimental to CODS. The main findings were trivial non-significant differences were observed for SLH and SLTH between left and right limbs (Table 1), however small significant differences were revealed between D and ND limbs (Table 1). In addition, directional dominance was also observed for CODS performance (Table 1); in agreement with our hypotheses. Contrary to our hypotheses, the size of imbalance had no detrimental impact on CODS, with no significant associations observed between hop imbalances and CODS (Table 2). Furthermore, hop imbalances were not significantly related to imbalances in CODS indicating that collegiate athletes who display greater hop asymmetries do not display greater CODS asymmetries (Table $2 \& 3$; Figure 1); in contrast to our hypotheses. Similarly, there was poor agreements of like for like identifications of asymmetry between hop performance and CODS, suggesting asymmetries in hopping do not necessarily correspond to a directional dominance during $180^{\circ}$ turns and $90^{\circ}$ cuts (Table 3; Figure 1).

The present study revealed trivial non-significant differences right and left limbs in hop performance, however small significant differences were found between D and ND limbs (Table 1), consistent with previous research in university, ${ }^{12}$ netball, ${ }^{13}$ and softball athletes ${ }^{27}$. Interestingly, the D hopping limb did not always correspond to throwing hand or kicking leg dominance as illustrated in Figures 1; similar to corroborative research. ${ }^{12}$ This could be due to specific sporting demands which may result in imbalances between limbs, such as the support limb during kicking a football or decelerating limb during a batting stroke and cricket bowl. ${ }^{27,32}$

Contrary to expectations, hop imbalances had no detrimental impact on turning and cutting performance (Table 2) which supports the only other study to investigate the impact of between limb asymmetries in unilateral hops on CODS perfor- 
mance reporting no effect. ${ }^{10}$ This outcome is in contrast to previous research which has shown imbalances in unilateral drop jump height and reactive strength associated with slower cutting performance. ${ }^{7,8}$ Conversely, no detrimental effect of imbalances in unilateral vertical jump power between $\mathrm{D}$ and ND limbs for a three cone CODS task was revealed. ${ }^{11}$ While, mixed results have been reported, with faster athletes demonstrating significantly greater imbalances in knee extensor torque $\left(240^{\circ}\right)$ but lower imbalances in eccentric knee flexor torque $\left(30^{\circ}\right)$ compared to slower athletes. ${ }^{9}$ The lack of consensus regarding the impact of imbalances on CODS could be attributed to differences in the methods to assess imbalance, CODS tasks, angle of direction change, asymmetry calculation, statistical analysis procedures and athlete populations.

Directional dominance was observed in the present study (Table 1), similar to corroborative research. ${ }^{8,23}$ Notably, eight and nine subjects demonstrated imbalances greater than asymmetry thresholds -2.74 and $-4.93 \%$ for $\bmod 505$ and $90^{\circ}$ cut performance, respectively (Table 3). Surprisingly, athletes with greater asymmetries in hopping performance did not exhibit greater imbalances between directions for both mod505 and $90^{\circ}$ cutting CODS tasks (Table 2; Figure 1). Moreover, low percentage agreements were observed between like for like identifications of hop and CODS performance suggesting that a D limb in hopping does not necessarily correspond to faster performance from that push off limb during cutting and pivot CODS tasks (Table 3, Figure 1). This discrepancy may be attributed to the subtle differences in the orientation of force application suggesting that an asymmetry in sagittal plane force application (hop distance) may not equate to asymmetry in frontal plane force application (cutting), thus supporting the notion that asymmetries are task specific. ${ }^{12}$ Furthermore, CODS is constituted by multifactorial variables such as technique for deceleration or re-acceleration (i.e., foot placement, adjustment of strides, body lean and posture), straight sprint speed and lower limb strength and power qualities. ${ }^{8,20}$ Thus CODS performance in the present study may involve the multifaceted influence other than limbs asymmetries in hopping, such as asymmetries in eccentric strength, ${ }^{9}$ lower limb stiffness ${ }^{7}$ or reactive strength $^{8}$.

Specifically, eight subjects were classified as asymmetrical (SLH) with an imbalance exceeding 7.25\% (Table 3; Figure 1), however only two of those subjects demonstrated directional dominance from that same limb for mod505 performance; and only one subject corresponded to faster performance for cutting (Figure 1). Similarly, 10 subjects were classified as asymmetrical in SLTH, but only three subjects demonstrated asymmetries which corresponded to the directional dominance for mod505, and two subjects corresponded to cutting performance (Table 3; Figure 1). Therefore, it can be inferred that a D hopping limb may not necessarily correspond to faster performance from that push off limb during a mod505 and $90^{\circ}$ cut (Table 3; Figure 1). This data refutes the observations of previous work who reported a trend in reactive strength dominance corresponding to faster performance from that limb during cuts $\left(20-60^{\circ}\right) .{ }^{8}$
Poor like for like identifications of asymmetry for cutting and pivoting CODS were demonstrated (32\%) (Table 3), indicating that the push off limb responsible for the superior cutting performance may not necessarily equate to superior turning performance from that same limb, and vice versa. Figures $1 \& 2$ illustrate the individual variation between directional dominance for cutting and pivoting CODS tasks. This data refutes the preconceived notion that superior performance from one change of direction task transfers to another and supports the concept that the biomechanical demands of change of direction are angle dependant and as such are independent skills. ${ }^{33-35}$

Significant mean differences were observed between D and ND CODS, with greater imbalances observed between cutting directions compared to mod505 directions (Table 1). Irrespective of direction, theoretically the time taken to enter the change of direction should be similar between directions thus, the imbalance and deficit in completion time suggests there is a movement deficiency in changing direction to the ND side. Alternatively, bilateral differences in joint-joint coordination on approach and re-acceleration leading to technical differences between sides, along with modifications of approach velocity may explain the longer completion time to the ND direction. Further research is required investigating the kinematic and kinetic differences between directions during $180^{\circ}$ turning and $90^{\circ}$ cutting CODS performance.

$A \geq 10-15 \%$ imbalance has been stated to represent a problematic asymmetry. ${ }^{1}$ The imbalances observed in the present study for mod505 and cutting performance are representative of dynamic tasks (Table 1), closely related to sporting movements, but fall below the suggested problematic criteria. The imbalances although not exceeding the $10-15 \%$ value, could still be interpreted as a deficiency in movement, and potentially problematic in multidirectional sports where it would be advantageous to be equally proficient in changing direction effectively off both limbs due to the unpredictable nature of the sport. Consequently, practitioners are recommended to inspect both directions in CODS testing batteries, firstly to eliminate bias to athletes with directional dominance when examining only one direction; ${ }^{23}$ secondly, to identify any imbalances in completion time between sides which could be indicative of a deficiency in change of direction ability to a direction. ${ }^{23}$

The results from the present study propose there is a no detrimental impact of asymmetries in hop performance to CODS and athletes with greater asymmetries in hops do not display superior turning and cutting from that D hopping limb. However, with a mixed small sample size and low number of subjects displaying hop imbalances greater than $10-15 \%$ suggested problematic criteria (Figures 1), caution must be applied when interpreting these findings. For example, only three and five subjects displayed imbalances which exceeded $10 \%$ for SLH and SLTH, respectively, while only two subjects displayed imbalances which exceeded $15 \%$ for SLH only (Figures 1). Thus, the imbalances observed (small) may not have been high enough to elicit detriments in CODS, however the lack of effect of hop asymmetries on CODS is consistent 
with the only other investigation to explore this. ${ }^{10}$ Furthermore, no general consensus exists for an asymmetry threshold cut-off in the literature; the present study used the mean imbalance $+(0.2 S D)$ asymmetry threshold used previously, ${ }^{10}$ but greater agreements may be observed with more conservative criteria such as mean $+(1 S D)^{24}$.

Another note of caution is the present study only investigated hopping tasks in the sagittal plane with a unilateral landing, although further hop distances may been achieved with a bilateral landing and investigation of a lateral jump could have greater specificity to cutting due to the medio-lateral force requirements of cutting. However, notably no differences in 505 and t-test performance between athletes of higher and lower levels of lateral jump asymmetry were reported. ${ }^{10}$ Therefore, the lack of significant correlations between imbalances and CODS (Table 2), and poor percentage agreements between like for like identifications of asymmetry (Table 3) indicate that athletes with hop imbalances within the range reported in this study (Table 1; Figure 1) should not experience associated detriments in mod505 and $90^{\circ}$ cutting performance.

\section{CONCLUSION}

Collegiate athletes display directional dominance during $\bmod 505$ and $90^{\circ}$ cutting performance; therefore, practitioners are encouraged to assess both directions when assessing CODS in their athletes to eliminate bias and to identify performance deficits between directions. Greater asymmetries could be interpreted as a deficiency in change of direction ability. The SLH and SLTH are indirect assessments of horizontal (forward) propulsion and force acceptance, and produce reliable measures of hop distance. Small significant differences between D and ND limbs were observed for hop performance in collegiate male athletes, but asymmetries in hop distance had no effect on CODS. Therefore, athletes with hop imbalances less than the imbalances reported in the present study should not experience detriments to COD. Furthermore, the D limb for hop performance does not necessarily correspond to faster performance from that limb during $180^{\circ}$ turns and $90^{\circ}$ cuts (push off limb).

\section{ACKNOWLEDGMENTS}

The authors would like to thank the individuals who participated in this investigation. No grant funding was received to support this research and the authors have no conflict of interest.

\section{REFERENCES}

1. Kannus P. Isokinetic evaluation of muscular performance: implications for muscle testing and rehabilitation. Int J Sports Med 1994;15:S11-S18.

2. Nadler SF, Malanga GA, Feinberg JH, Prybicien M, Stitik TP, DePrince M. Relationship between hip muscle imbalance and occurrence of low back pain in collegiate athletes: a prospective study. Am J Phys Med Rehab 2001;80:572-577.

3. Kiesel K, Plisky PJ, Voight ML. Can serious injury in professional football be predicted by a preseason functional movement screen. $N$ Am J Sports Phys Ther 2007;2:147-158.

4. Knapik JJ, Bauman CL, Jones BH, Harris JM, Vaughan L. Preseason strength and flexibility imbalances associated with athletic injuries in female collegiate athletes. Am J Sports Med 1991;19:76-81.

5. Bennell K, Wajswelner H, Lew P, Schall-Riaucour A, Leslie S, Plant D, et al. Isokinetic strength testing does not predict hamstring injury in Australian Rules footballers. Brit J Sport Med 1998;32:309-314.

6. Beukeboom C, Birmingham TB, Forwell L, Ohrling D. Asymmetrical strength changes and injuries in athletes training on a small radius curve indoor track. Clin J Sport Med 2000;10:245-250.

7. Maloney SJ, Richards J, Nixon DG, Harvey LJ, Fletcher IM. Do stiffness and asymmetries predict change of direction performance? J Sports Sci 2017;35:547-556.

8. Young WB, James R, Montgomery I. Is muscle power related to running speed with changes of direction? J Sport Med Phys Fitness 2002;42:282288.

9. Lockie RG, Schultz AB, Jeffriess MD, Callaghan SJ. The relationship between bilateral differences of knee flexor and extensor isokinetic strength and multi-directional speed. Isokinet Exerc Sci 2012;20:211-219.

10. Lockie RG, Callaghan SJ, Berry SP, Cooke ER, Jordan CA, Luczo TM, et al. Relationship between unilateral jumping ability and asymmetry on multidirectional speed in team-sport athletes. J Strength Cond Res 2014; 28:3557-3566.

11. Hoffman JR, Ratamess NA, Klatt M, Faigenbaum AD, Kang J. Do bilateral power deficits influence direction-specific movement patterns? Res Sports Med 2007; 15:125-132.

12. Jones PA, Bampouras TM. A comparison of isokinetic and functional methods of assessing bilateral strength imbalance. J Strength Cond Res 2010;24:1553-1558.

13. Hewit JK, Cronin JB, Hume PA. Asymmetry in multi-directional jumping tasks. Phys Ther Sport 2012;13:238-242.

14. Bell DR, Sanfilippo JL, Binkley N, Heiderscheit BC. Lean mass asymmetry influences force and power asymmetry during jumping in collegiate athletes. $J$ Strength Cond Res 2014;28:884-891.

15. Dos'Santos T, Thomas C, Jones PA, Comfort P. Assessing muscle strength asymmetry via a unilateral stance isometric mid-thigh pull. Int $J$ Sports Physiol Perform 2016, in press.

16. Stolberg M, Sharp A, Comtois AS, Lloyd RS, Oliver JL, Cronin J. Triple and Quintuple Hops: Utility, Reliability, Asymmetry, and Relationship to Performance. Strength Cond J 2016;38:18-25.

17. Munro AG, Herrington LC. Between-session reliability of four hop tests and the agility T-test. J Strength Cond Res 2011;25:1470-1477.

18. Meylan, McMaster T, Cronin J, Mohammad NI, Rogers C. Single-leg lateral, horizontal, and vertical jump assessment: reliability, interrelationships, and ability to predict sprint and change-of-direction performance. J Strength Cond Res 2009;23:1140-1147.

19. Hamilton RT, Shultz SJ, Schmitz RJ, Perrin DH. Triple-hop distance as a valid predictor of lower limb strength and power. J Athl Training 2008; 43:144-151.

20. Sheppard JM, Young WB. Agility literature review: classifications, training and testing. J Sports Sci 2006;24:919-932.

21. Sheppard JM, Young WB, Doyle TLA, Sheppard TA, Newton RU. An evaluation of a new test of reactive agility and its relationship to sprint speed and change of direction speed. J Sci Med Sport 2006;9:342-349.

22. Negrete R, Brophy J. The relationship between isokinetic open and closed chain lower extremity strength and functional performance. J Sport Rehabil 2000;9:46-61.

23. Hart NH, Spiteri T, Lockie RG, Nimphius S, Newton RU. Detecting deficits in change of direction performance using the preplanned multidirectional Australian Football League agility test. $J$ Strength Cond Res 2014;28:3552-3556.

24. Graham-Smith P, Al-Dukhail A, Jones P, editors. Agreement between attributes associated with bilateral jump asymmetry. ISBS-Conference Proceedings Archive; 2016.

25. Faul F, Erdfelder E, Buchner A, Lang A-G. Statistical power analyses using $\mathrm{G}^{*}$ Power 3.1: Tests for correlation and regression analyses. Behav Res Methods 2009;41:1149-1160.

26. Thomas C, Comfort P, Chiang C-Y, Jones P. Relationship between 
isometric mid-thigh pull variables and sprint and change of direction performance in collegiate athletes. $J$ Trainol 2015;4:6-10.

27. Newton RU, Gerber A, Nimphius S, Shim JK, Doan BK, Robertson M, et al. Determination of functional strength imbalance of the lower extremities. J Strength Cond Res 2006;20:971-977.

28. Atkinson G, Nevill AM. Statistical methods for assessing measurement error (reliability) in variables relevant to sports medicine. Sports Med 1998;26:217-238.

29. Hedges L, Olkin I. Statistical Methods for Meta-Analysis. New York: Acdemic Press; 1985.

30. Hopkins WG. A scale of magnitudes for effect statistics. A new view of statistics. 2002.
31. Hopkins WG. Measures of reliability in sports medicine and science. Sports Med 2000;30:1-15.

32. Gabbard C, Hart S. A question of foot dominance. J Gen Psychol 1996; 123:289-296.

33. Jones PA, Herrington L, Graham-Smith P. Braking characteristics during cutting and pivoting in female soccer players. J Electromyogr Kines 2016; 30:46-54.

34. Havens KL, Sigward SM. Whole body mechanics differ among running and cutting maneuvers in skilled athletes. Gait Posture 2014;42:240-245.

35. Cortes N, Onate J, Van Lunen B. Pivot task increases knee frontal plane loading compared with sidestep and drop-jump. J Sports Sci 2011;29:8392 . 